\title{
Combination of Methotrexate and Leflunomide for Adult-onset Still's Disease: A Case Report and Literature Review
}

\author{
Eunyoung Emily Lee, M.D., Min Jung Kim, M.D., Yeong Wook Song, M.D., Ph.D., Jin Kyun Park, M.D., Ph.D. \\ Division of Rheumatology, Department of Internal Medicine, Seoul National University Hospital, Seoul, Korea
}

The treatment of adult-onset Still's disease (AOSD) aims to control systemic inflammation and prevent organ damage. Systemic inflammation can be controlled with corticosteroid (CS) monotherapy in most cases. However, symptoms often flare as CS is tapered, often requiring long-term CS treatment, with its associated risks of infection, cardiovascular disease, and osteoporosis. Disease-modifying antirheumatic drugs (DMARDs) are often used as CS-sparing agents; however, the choice of DMARD has been largely empirical. Methotrexate (MTX) is recommended as the first-line steroid-sparing drug due to its well-known efficacy and safety in rheumatoid arthritis (RA). When MTX treatment is unsuccessful in AOSD, the choice of a second-line drug has not been established. In RA, leflunomide (LEF) has been used as an alternative to or in combination with MTX. To date, there has been no adequate assessment of the combination of LEF and MTX in AOSD. Here, we report a case of refractory chronic AOSD successfully treated with the MTX-LEF combination. (J Rheum Dis 2020;27:116-119)

Key Words. Still's disease, Adult-onset, Antirheumatic agents, DMARD, Methotrexate, Leflunomide

\section{INTRODUCTION}

Adult-onset Still's disease (AOSD) is a rare systemic inflammatory disease of unknown etiology that manifests as a spiking fever, arthritis, and an evanescent rash, along with diffuse lymphadenopathy, splenomegaly, and high ferritin levels. The treatment of AOSD aims to control the systemic inflammation and prevent organ damage. In the absence of life-threatening complications, such as macrophage activation syndrome (MAS) and acute respiratory distress syndrome, the systemic inflammation of AOSD can be controlled with corticosteroids (CS) in most cases [1]. However, symptoms often flare when CS is tapered off, and a subset of patients require long-term CS therapy, which is associated with an increased risk of infection, cardiovascular disease, and osteoporosis [2]. Conventional or biologic disease-modifying antirheumatic drugs (DMARDs) are recommended as CS-sparing agents [3].
In the absence of randomized clinical trials, the choice of DMARD has been largely empirical. Methotrexate (MTX) is recommended as the first-line steroid-sparing drug due to its efficacy and well-known safety profile established in rheumatoid arthritis (RA) patients [1,4]. However, when MTX therapy is unsuccessful, a second-line drug of choice has not yet been established. In patients with RA, leflunomide (LEF) has been successfully used in combination with MTX, demonstrating a favorable efficacy and safety profile. To date, there has been no adequate assessment of combined LEF and MTX treatment in AOSD. Here, we describe a case of refractory AOSD that was successfully treated with the MTX-LEF combination.

The study was approved by the institutional review board of the Seoul National University Hospital (IRB No: 1802-120-924). The requirement for informed consent was waived as this case report involved minimal risk to the patients and no identifiable information was use.

Received : February 6, 2020, Revised : March 11, 2020, Accepted : March 12, 2020

Corresponding to : Jin Kyun Park iD http://orcid.org/0000-0003-2167-9393

Division of Rheumatology, Department of Internal Medicine, Seoul National University Hospital, 101 Daehak-ro, Jongno-gu, Seoul 03080, Korea. E-mail : jinkyunpark@gmail.com

Copyright (c) 2020 by The Korean College of Rheumatology. All rights reserved.

This is an Open Access article, which permits unrestricted non-commerical use, distribution, and reproduction in any medium, provided the original work is properly cited. 


\section{CASE REPORT}

A 42-year-old man with a history of acute rheumatic fever as a child presented with fever for 3 weeks. The patient also complained of chest pain, sore throat, and arthralgia. Electrocardiogram (ECG) showed an ST elevation in leads $\mathrm{V} 1$ and $\mathrm{V} 2$, and the troponin I level was $0.06 \mathrm{ng} / \mathrm{mL}$ (reference range, $0 \sim 0.028 \mathrm{ng} / \mathrm{mL}$ ). Echocardiography showed mild septal bouncing, suggesting an early pericarditis with constrictive physiology. Nonsteroidal anti-inflammatory drugs were initiated to manage the acute pericarditis and chest pain. Although the ST elevation on ECG was resolved, the fever and arthralgia persisted. The fever showed a diurnal pattern, with the body temperature reaching $40^{\circ} \mathrm{C}$ in the afternoon. He had no skin rash. The white blood cell count was 32,310/ $\mu \mathrm{L}$, the high-sensitivity C-reactive protein level was $79.8 \mathrm{mg} / \mathrm{L}$, and the erythrocyte sedimentation rate was $43 \mathrm{~mm} / \mathrm{h}$. Aspartate transaminase (AST) and alanine transaminase (ALT) levels were elevated to $132 \mathrm{IU} / \mathrm{L}$ and $112 \mathrm{IU} / \mathrm{L}$, respectively, and the serum ferritin level was 139,599 $\mathrm{ng} / \mathrm{mL}$. Tests for anti-streptolysin $\mathrm{O}$ titers, anti-nuclear antibodies (ANA), and rheumatoid factor (RF) were negative. The blood culture was negative. Computed tomography of the chest and abdomen showed diffuse lymphadenopathy and splenomegaly. Bone marrow biopsy showed a reactive marrow with no evidence of malignancy. On the basis of Yamaguchi's criteria of fever, arthralgia, leukocytosis, sore throat, lymphadenopathy, splenomegaly, elevated AST and ALT, and negative ANA and RF, AOSD was diagnosed. A single dose of methylprednisolone (125 mg) was administered, resulting in prompt symptom improvement. The patient's condition was sub- sequently managed with prednisolone (PD) at $60 \mathrm{mg} /$ day and MTX at $15 \mathrm{mg} /$ week. However, after 10 weeks, when the PD was tapered to $20 \mathrm{mg} /$ day, the diurnal spiking fever returned, with high expression of inflammatory markers, suggestive of a flare. An influenza test was negative. There was no evidence of infection. As the patient was unable to taper PD to below $10 \mathrm{mg}$ /day, additional DMARD therapy was felt to be necessary. Since there was no evidence of life-threatening conditions such as MAS, LEF was administered with the MTX instead of tocilizumab or anakinra (Figure 1). Clinical symptoms and laboratory parameters improved. The PD was slowly tapered off, and the patient remained in remission.

\section{DISCUSSION}

A review of the literature reveals three case reports where LEF was used in combination with other DMARDs to treat refractory AOSD (Table 1) [5-7]. In the first report, a patient with chronic AOSD relapsed repeatedly during the CS taper, despite receiving a combination of hydroxychloroquine (200 mg/day), MTX (25 mg/week), and azathioprine $(2.5 \mathrm{mg} / \mathrm{kg} /$ day $)$ or intravenous cyclophosphamide $(750 \mathrm{mg} /$ day) every 4 weeks. In this refractory patient, adding LEF to azathioprine successfully controlled the disease [5]. In the second case report, the LEF and chloroquine combination was successful in two patients [6]. In the third report, AOSD symptoms returned when PD was tapered to below $20 \mathrm{mg}$ /day despite concurrent use of MTX (15 mg/week) and sulfasalazine (2,000 $\mathrm{mg} /$ day). The patient achieved remission when sulfasalazine was replaced with LEF [7]. In another case report, an AOSD patient who failed to respond to the PD,

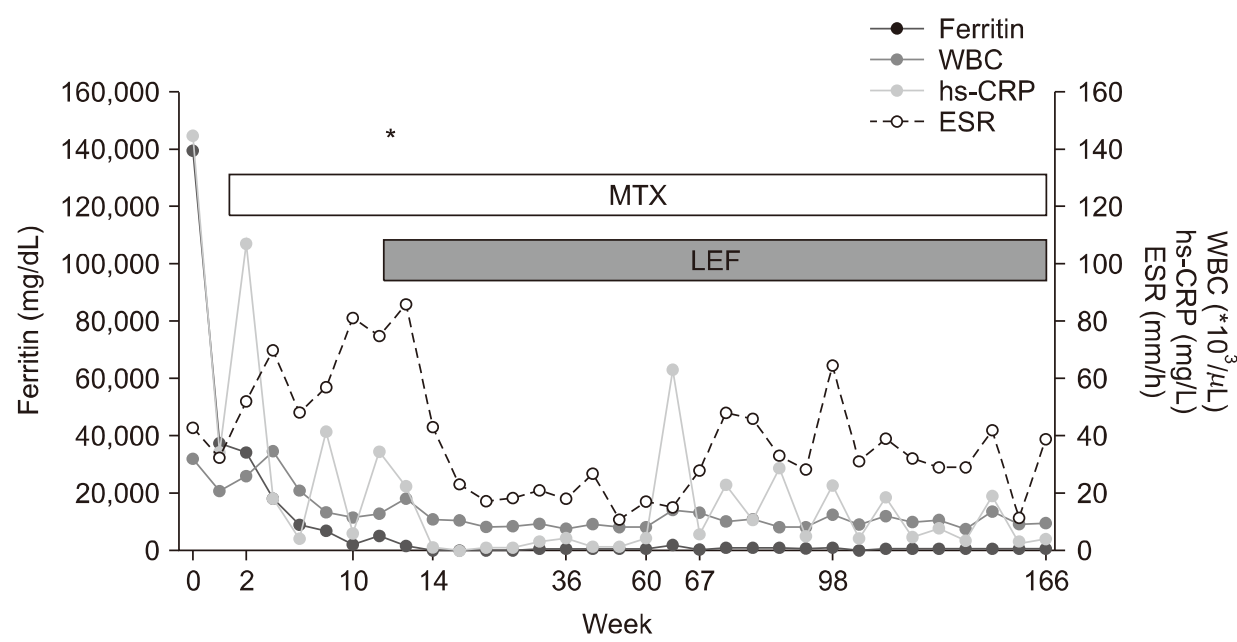

Figure 1. Changes in laboratory test results of the patient after a diagnosis of AOSD. Asterisk represents flare. AOSD: adultonset Still's disease, ESR: erythrocyte sedimentation rate, hsCRP: high sensitivity C-reactive protein, WBC: white blood cell, LEF: leflunomide, MTX: methotrexate. 
Eunyoung Emily Lee et al.

Table 1. Summary of the literature

\begin{tabular}{|c|c|c|c|c|c|}
\hline Publication & $\begin{array}{l}\text { No. of } \\
\text { cases }\end{array}$ & Age/Sex & Clinical features & Treatment prior to LEF & $\begin{array}{l}\text { Treatment with } \\
\text { remission }\end{array}$ \\
\hline Present report & 1 & 42/male & $\begin{array}{l}\text { Fever, arthralgia, } \\
\text { pericarditis, sore throat, } \\
\text { lymphadenopathy, } \\
\text { splenomegaly }\end{array}$ & $\begin{array}{l}\text { PD } 60 \mathrm{mg} \rightarrow 20 \mathrm{mg} / \text { day, } \\
\text { MTX }\end{array}$ & MTX + LEF \\
\hline Cefle et al., 2005 [5] & 1 & 24/female & $\begin{array}{l}\text { Fever, arthritis, sore throat, } \\
\text { rash, hepatosplenomegaly, } \\
\text { lymphadenopathy }\end{array}$ & $\begin{array}{l}\text { mPD 1g/day for } 3 \text { days, } \\
\text { indomethacin, HCQ, } \\
\text { MTX, AZA, IV CTX }\end{array}$ & $\mathrm{AZA}+\mathrm{LEF}$ \\
\hline Pirildar et al., 2003 [6] & 2 & & Pericardial effusion & CS & Chloroquine + LEF \\
\hline Sayarlioglu et al., 2007 [7] & 1 & 19/male & $\begin{array}{l}\text { Fever, rash, sore throat, } \\
\text { polyarthralgia/arthritis, } \\
\text { hepatomegaly }\end{array}$ & $\begin{array}{l}\text { PD } 1 \text { mg/kg/day, MTX, } \\
\text { indomethacin, } \\
\text { sulfasalazine }\end{array}$ & MTX + LEF \\
\hline
\end{tabular}

LEF: leflunomide, PD: prednisolone, mPD: methylprednisolone, HCQ: hydroxychloroquine, MTX: methotrexate, AZA: azathioprine, IV: intravenous, CTX: cyclophosphamide, CS: corticosteroid.

MTX, and hydroxychloroquine combination was treated with MTX-LEF [8].

It has been reported that up to $75 \%$ of patients with AOSD relapse and have a chronic disease course [9], requiring high cumulative doses of CSs. However, in the absence of randomized trials, steroid-sparing strategies for patients with relapsing AOSD are largely based on empirical evidence. LEF is an isoxazole derivative with immunomodulatory and disease-modifying properties. The immunomodulatory effect is achieved by inhibiting the mitochondrial enzyme dihydroorotate dehydrogenase, which is critical for the de novo synthesis of pyrimidines, which are required for the synthesis of DNA and RNA [10]. Inhibition of this enzyme prevents lymphocyte activation and clonal expansion of T lymphocytes [11]. In addition, LEF is known to inhibit the expression of proinflammatory cytokines such as tumor necrosis factor alpha and interleukin (IL)-1 $\beta$ [12]. The combination of MTX and LEF in RA reduced serum levels of IL-17 [13] and resulted in better clinical outcomes than MTX alone, with a comparable efficacy to a biological DMARD [14]. For this reason, we chose to administer LEF along with MTX in this patient, even though the time of onset of action is expected to be slower for LEF than for the IL-6 inhibitor tocilizumab.

An emerging body of evidence supports the early use of treatments directly targeting inflammatory cytokines in AOSD. However, those biologic DMARDs are associated with an increased risk of infection and drug hypersensitivity reactions [15]. Also, some patients prefer oral therapy, especially when long-term treatment is required.
Thus, the MTX-LEF combination could be effective in patients who fail to taper CS with conventional DMARD monotherapy and who prefer oral medication. However, there are reports of cases refractory to the MTX-LEF combination. In addition, it is important to emphasize that administration of biologic DMARDs with a rapid onset of action, such as tocilizumab and anakinra, should not be delayed in acute, life-threatening situations, given the slow onset of action of MTX and LEF [1]. Once life-threatening danger is averted, tocilizumab can be replaced with MTX and/or LEF.

In our case, the combination of MTX and LEF not only controlled systemic inflammation and articular symptoms, but also effectively prevented relapse. The patient tolerated the MTX-LEF combination well, and no treatment-associated adverse events such as hepatotoxicity were observed.

\section{SUMMARY}

The MTX-LEF combination represents a possible alternative approach in patients with refractory chronic AOSD, who flare during CS taper. Further prospective studies are required to evaluate the efficacy and safety of the MTX-LEF combination in AOSD.

\section{CONFLICT OF INTEREST}

No potential conflict of interest relevant to this article was reported. 


\section{AUTHOR CONTRIBUTIONS}

E.E.L. and J.K.P. were in charge of design of the study, acquisition of data and analysis and interpretation of data. E.E.L. wrote the manuscript and prepared tables and figures. M.J.K., Y.W.S. and J.K.P. gave critical revision of the manuscript. All authors read and approved the final manuscript.

\section{REFERENCES}

1. Govoni M, Bortoluzzi A, Rossi D, Modena V. How I treat patients with adult onset Still's disease in clinical practice. Autoimmun Rev 2017;16:1016-23.

2. van der Goes MC, Jacobs JW, Bijlsma JW. The value of glucocorticoid co-therapy in different rheumatic diseases--positive and adverse effects. Arthritis Res Ther 2014;16 Suppl 2:S2.

3. Mimura T, Kondo Y, Ohta A, Iwamoto M, Ota A, Okamoto $\mathrm{N}$, et al. Evidence-based clinical practice guideline for adult Still's disease. Mod Rheumatol 2018;28:736-57.

4. Fautrel B, Borget C, Rozenberg S, Meyer O, Le Loët X, Masson C, et al. Corticosteroid sparing effect of low dose methotrexate treatment in adult Still's disease. J Rheumatol 1999;26:373-8.

5. Cefle A. Leflunomide and azathioprine combination in refractory adult-onset Still's disease. Ann Pharmacother 2005;39:764-7.

6. Pirildar T. Treatment of adult-onset Still's disease with leflunomide and chloroquine combination in two patients. Clin Rheumatol 2003;22:157.
7. Sayarlioglu M, Ucmak H, Ozkaya M, Ucar MA, Sayarlioglu $\mathrm{H}$. Leflunomide and methotrexate combination in refractory adult-onset Still's disease. Ann Pharmacother 2007;41:1319-20.

8. Sayarlioglu M, Sahin M, Cetin GY, Avan R, Cerit M. Maternal exposure to leflunomide and methotrexate in a patient with adult-onset Still's disease. Rheumatology (Oxford) 2010;49:1787-9.

9. Kong XD, Xu D, Zhang W, Zhao Y, Zeng X, Zhang F. Clinical features and prognosis in adult-onset Still's disease: a study of 104 cases. Clin Rheumatol 2010;29:1015-9.

10. Teschner S, Burst V. Leflunomide: a drug with a potential beyond rheumatology. Immunotherapy 2010;2:637-50.

11. Cherwinski HM, McCarley D, Schatzman R, Devens B, Ransom JT. The immunosuppressant leflunomide inhibits lymphocyte progression through cell cycle by a novel mechanism. J Pharmacol Exp Ther 1995;272:460-8.

12. Hochberg MC, Silman AJ, Smolen JS, Weinblatt ME, Weisman MH. Rheumatology. 5th ed. Philadelphia, Mosby/ Elsevier, 2011.

13. Yao Y, Ding CZ, Fang Y. Combination of MTX and LEF attenuates inflammatory bone erosion by down-regulation of receptor activator of NF-kB ligand and interleukin-17 in type II collagen-induced arthritis rats. Rheumatol Int 2013; 33:1845-53.

14. Kremer JM, Genovese MC, Cannon GW, Caldwell JR, Cush JJ, Furst DE, et al. Concomitant leflunomide therapy in patients with active rheumatoid arthritis despite stable doses of methotrexate. A randomized, double-blind, placebo-controlled trial. Ann Intern Med 2002;137:726-33.

15. Al-Homood IA. Biologic treatments for adult-onset Still's disease. Rheumatology (Oxford) 2014;53:32-8. 